DSF-16/2005, IFIC/05-17, MPP-2005-36

\title{
Relic neutrino decoupling including flavour oscillations
}

\author{
Gianpiero Mangano ${ }^{\mathrm{a}, \mathrm{b}}$, Gennaro Miele ${ }^{\mathrm{a}}$, Sergio Pastor ${ }^{\mathrm{c}}$, \\ Teguayco Pinto $^{c}$, Ofelia Pisanti ${ }^{a}$, Pasquale D. Serpico ${ }^{d}$ \\ ${ }^{a}$ Dipartimento di Scienze Fisiche, Università di Napoli Federico II and INFN, \\ Sezione di Napoli, Complesso Universitario di Monte S. Angelo, Via Cintia, \\ I-80126 Naples, Italy \\ b Department of Physics, Syracuse University, Syracuse NY, 13244-1130, USA \\ ${ }^{\mathrm{c}}$ Instituto de Física Corpuscular (CSIC-Universitat de València), Ed. Institutos de \\ Investigación, Apdo. 22085, E-46071 Valencia, Spain \\ ${ }^{\mathrm{d}}$ Max-Planck-Institut für Physik (Werner-Heisenberg-Institut), Föhringer Ring 6, \\ D-80805 Munich, Germany
}

\begin{abstract}
In the early universe, neutrinos are slightly coupled when electron-positron pairs annihilate transferring their entropy to photons. This process originates non-thermal distortions on the neutrino spectra which depend on neutrino flavour, larger for $\nu_{e}$ than for $\nu_{\mu}$ or $\nu_{\tau}$. We study the effect of three-neutrino flavour oscillations on the process of neutrino decoupling by solving the momentum-dependent kinetic equations for the neutrino spectra. We find that oscillations do not essentially modify the total change in the neutrino energy density, giving $N_{\text {eff }}=3.046$ in terms of the effective number of neutrinos, while the small effect over the production of primordial ${ }^{4} \mathrm{He}$ is increased by $\mathcal{O}(20 \%)$, up to $2.1 \times 10^{-4}$. These results are stable within the presently favoured region of neutrino mixing parameters.
\end{abstract}

Key words: Early Universe; Neutrinos; Non-equilibrium kinetics

\section{Introduction}

The existence of a relic sea of neutrinos is a generic prediction of the standard hot big bang model, in number only slightly below that of relic photons that constitute the Cosmic Microwave Background (CMB). The presence of the cosmic neutrino background has been indirectly established both at

Preprint submitted to Elsevier Science 2 February 2008 
$\mathrm{MeV}$ temperatures by the accurate agreement between the calculated and observed primordial abundances of light elements from Big Bang Nucleosynthesis (BBN) and at later epochs by data on the anisotropies of the CMB and the distribution of Large Scale Structures (LSS) in the universe, through the contribution of neutrinos to the radiation content.

These cosmic neutrinos were kept in equilibrium by frequent weak interactions with other particles until the temperature of the Universe was of order 2-5 $\mathrm{MeV}$, when these interactions became ineffective and the process of neutrino decoupling took place. The standard picture in the instantaneous decoupling limit is very simple (see e.g. [1]): coupled neutrinos had a momentum spectrum with an equilibrium Fermi-Dirac (FD) form with temperature $T$,

$$
f_{\mathrm{eq}}(p)=\left[\exp \left(\frac{p-\mu_{\nu}}{T}\right)+1\right]^{-1}
$$

which is preserved after decoupling, since both neutrino momenta and temperature redshift identically with the universe expansion. Here we have included a potential neutrino chemical potential $\mu_{\nu}$ that would exist in the presence of a neutrino-antineutrino asymmetry, but it was shown in [2] that the stringent BBN bounds on $\mu_{\nu_{e}}$ apply to all flavours, since neutrino oscillations lead to flavour equilibrium before BBN. Thus the contribution of a relic neutrino asymmetry can be safely ignored.

Shortly after neutrino decoupling the photon temperature drops below the electron mass, favouring $e^{ \pm}$annihilations that heat the photons. If one assumes that this entropy transfer did not affect the neutrinos because they were already completely decoupled, it is easy to calculate the difference between the temperatures of relic photons and neutrinos $T_{\gamma} / T_{\nu}=(11 / 4)^{1 / 3} \simeq 1.40102$. However, the processes of neutrino decoupling and $e^{ \pm}$annihilations are sufficiently close in time so that some relic interactions between $e^{ \pm}$and neutrinos exist. These relic processes are more efficient for larger neutrino energies, leading to non-thermal distortions in the neutrino spectra and a slightly smaller increase of the comoving photon temperature, as noted in previous works (for early references, see [3] and the full list given in the review [4]).

A proper calculation of the process of non-instantaneous neutrino decoupling demands solving the momentum-dependent Boltzmann equations for the neutrino spectra, a set of integro-differential kinetic equations that are difficult to solve numerically. In the early 1990s several works $[5,6,7]$ performed momentum-dependent calculations assuming some approximations, such as Boltzmann statistics for neutrinos, while the full numerical computation was later carried out in refs. [8,9,10,11]. Finally, a further refinement involves the inclusion of finite temperature QED corrections to the electromagnetic plasma, as done in $[12,13]$. 
The distortions produced on the neutrino momentum distributions are small and their direct observation is out of question. However, they should be included in a calculation of any observable related to relic neutrinos. For instance, non-thermal distortions lead to an enhanced number density of relic neutrinos which modifies e.g. the contribution of massive neutrinos to the present energy density of the universe. Previous analyses focused on two interesting effects. The first one concerns the contribution of neutrinos to the total radiation content of the universe, parametrized in terms of the effective number of neutrinos $[14,15] N_{\text {eff }}$, through the relation ${ }^{1}$

$$
\rho_{\mathrm{R}}=\left[1+\frac{7}{8}\left(\frac{4}{11}\right)^{4 / 3} N_{\mathrm{eff}}\right] \rho_{\gamma},
$$

where $\rho_{\gamma}$ is the energy density of photons, whose value today is known from the measurement of the CMB temperature. This equation holds as long as all neutrinos are relativistic and, in principle, $N_{\text {eff }}$ can receive a contribution from other relativistic relics. In the following we will restrict our analysis to the standard case, where the departure of $N_{\text {eff }}$ from 3 is due to neutrino heating by $e^{ \pm}$annihilations. The second effect of the non-thermal distortions leads to a modification of the outcome of $\mathrm{BBN}$, in particular a change in the production of primordial ${ }^{4} \mathrm{He}$. From previous works, an increase of order $1.5 \times 10^{-4}$ in the ${ }^{4} \mathrm{He}$ mass fraction $Y_{p}$ was found $[5,6,7,8,9,10,11]$. Thus the effect on BBN is small, but it has to be taken into account in precise BBN codes $[16,17]$.

In general, previous analyses of neutrino decoupling did not include flavour neutrino oscillations, although their potential effect was already noted long ago [18]. The exception is a work by Hannestad [19], who calculated the effect of two-neutrino oscillations on neutrino heating, finding that the neutrino energy density was slightly higher while the increase in the primordial abundance of ${ }^{4} \mathrm{He}$ due to non-thermal features of neutrino decoupling could be even doubled. In this work, some approximations were taken, such as integrated kinetic equations (quantum rate equations or QREs) and Maxwell-Boltzmann statistics. In addition, a very recent paper [20] considered the effects of neutrino oscillations in scenarios with low-reheating temperatures (below $10 \mathrm{MeV}$ ), using momentum-dependent equations with massless $e^{ \pm}$in the collision terms, but ignoring neutrino-neutrino collisions.

In the present paper we perform a new calculation of neutrino decoupling solving the momentum-dependent kinetic equations as in $[9,11,13]$, but including

1 This equation only holds after the reheating is completed, so it would be wrong if used in BBN calculations. For larger temperatures, one should modify it with $(4 / 11)^{4 / 3} \rightarrow\left(T_{\nu 0} / T_{\gamma 0}\right)^{4}$, where $T_{\nu 0} / T_{\gamma 0}$ traces the evolution derived just by the entropy conservation law, and add the contribution of $e^{ \pm}$while not completely nonrelativistic. 
also the effect of flavour neutrino oscillations following the analysis in [2]. Our aim is to compare our results with the simplified analysis in [19], checking the accuracy of its approximations. The numerical evaluation of the Boltzmann equations is computationally demanding, but we do not need to perform a scan over all the space of neutrino mixing parameters, since presently they are already known with good precision ${ }^{2}$ (except for the mixing angle $\theta_{13}$ which, as we will see, has a minor effect). We find that the results concerning neutrino decoupling remain unchanged within the presently favoured regions for oscillations parameters.

\section{Neutrino decoupling in presence of flavour oscillations}

In this section we list the set of equations which rule the process of neutrino decoupling in the epoch of the early universe prior to BBN. We describe the main terms that appear in the kinetic equations, in particular those arising from neutrino oscillations, and describe our method to solve these equations numerically.

\subsection{Equations}

In order to study neutrino decoupling in the early universe in the presence of flavour oscillations, we describe the neutrino ensemble in the usual way by generalized occupation numbers, i.e. by $3 \times 3$ density matrices for neutrinos and anti-neutrinos as described in $[25,26]$. The form of the neutrino density matrix for a mode with momentum $p$ is

$$
\varrho(p, t)=\left(\begin{array}{lll}
\varrho_{e e} & \varrho_{e \mu} & \varrho_{e \tau} \\
\varrho_{\mu e} & \varrho_{\mu \mu} & \varrho_{\mu \tau} \\
\varrho_{\tau e} & \varrho_{\tau \mu} & \varrho_{\tau \tau}
\end{array}\right)
$$

The diagonal elements correspond to the usual occupation numbers of the different flavours, while the off-diagonal terms are non-zero in the presence of neutrino mixing. There exists a corresponding set of equations for the antineutrino density matrix $\bar{\varrho}$, but in absence of a neutrino asymmetry it is not needed since antineutrinos follow the same the evolution as neutrinos.

2 Note that we focus on the standard case of three active neutrinos and do not consider active-sterile mixing (see instead e.g. refs. [21,22,23,24]). 
The equations of motion for the density matrices relevant for our situation of interest in an expanding universe are [25]

$$
i\left(\partial_{t}-H p \partial_{p}\right) \varrho_{p}=\left[\left(\frac{M^{2}}{2 p}-\frac{8 \sqrt{2} G_{\mathrm{F}} p}{3 m_{\mathrm{W}}^{2}} E\right), \varrho_{p}\right]+C\left[\varrho_{p}\right]
$$

where $H$ is the Hubble parameter, $G_{\mathrm{F}}$ is the Fermi constant and $m_{\mathrm{W}}$ the $W$ boson mass. We use the notation $\varrho_{p}=\varrho(p, t)$ and $[\cdot, \cdot]$ denotes the commutator. The vacuum oscillation term is proportional to $M^{2}$, the mass-squared matrix in the flavour basis that is related to the diagonal one in the mass basis $\operatorname{diag}\left(m_{1}^{2}, m_{2}^{2}, m_{3}^{2}\right)$ via the neutrino mixing matrix,

$$
\left(\begin{array}{ccc}
c_{12} c_{13} & s_{12} c_{13} & s_{13} \\
-s_{12} c_{23}-c_{12} s_{23} s_{13} & c_{12} c_{23}-s_{12} s_{23} s_{13} & s_{23} c_{13} \\
s_{12} s_{23}-c_{12} c_{23} s_{13} & -c_{12} s_{23}-s_{12} c_{23} s_{13} & c_{23} c_{13}
\end{array}\right) .
$$

Here $c_{i j}=\cos \theta_{i j}$ and $s_{i j}=\sin \theta_{i j}$ for $i j=12,23$, or 13 . Since we have assumed CP conservation, there are five oscillation parameters: $\Delta m_{21}^{2}=m_{2}^{2}-$ $m_{1}^{2}, \Delta m_{31}^{2}=m_{3}^{2}-m_{1}^{2}, \theta_{12}, \theta_{23}$ and $\theta_{13}$. From a global analysis of experimental data on flavour neutrino oscillations, the values of the first four parameters are determined, while we only have an upper bound on $\theta_{13}$. As a reference, we take the best-fit values from ref. [27],

$$
\left(\frac{\Delta m_{21}^{2}}{10^{-5} \mathrm{eV}^{2}}, \frac{\Delta m_{31}^{2}}{10^{-3} \mathrm{eV}^{2}}, s_{12}^{2}, s_{23}^{2}, s_{13}^{2}\right)=(8.1,2.2,0.3,0.5,0)
$$

while for $\theta_{13}$ we will also consider the value allowed at $3 \sigma, s_{13}^{2}=0.047$.

The decoupling of neutrinos takes place at temperatures of the order $\mathrm{MeV}$, when neutrinos experience both collisions and refractive effects from the medium. The latter correspond in Eq. (4) to the term proportional to the diagonal matrix $E$, that represents the energy densities of charged leptons. For example, $E_{e e}$ is the energy density of electrons and positrons. Note that we have neglected two terms in Eq. (4) with respect to the complete form shown in [25]. The first one is the usual refractive term $\sqrt{2} G_{\mathrm{F}} L$ that is proportional to the charged-lepton asymmetries. This asymmetric term is negligible at early times (high temperatures) compared to the $E$ term, while at temperatures near $n / p$ freeze out $(T \simeq 1 \mathrm{MeV})$ it is negligible compared to the vacuum term $M^{2} / 2 p$ for the mass-squared differences $\Delta m_{31}^{2}$ and $\Delta m_{21}^{2}$. The second refractive term not included in Eq. (4) arises from neutrino-neutrino interactions 
and is proportional ${ }^{3}$ to $(\varrho-\bar{\varrho})$, thus it vanishes for zero neutrino-antineutrino asymmetry.

Finally, the collisions of neutrinos with $e^{ \pm}$or among themselves are described by the term $C[\cdot]$, which is proportional to $G_{\mathrm{F}}^{2}$. For the off-diagonal complex terms of the density matrix we approximate collisions with a simple damping prescription of the form $C\left[\varrho_{\alpha \beta}(p)\right]=-D_{p} \varrho_{\alpha \beta}(p)$, with the same damping functions $D_{p}$ as in [2]. Instead, for the diagonal ones, in order to properly calculate the neutrino heating process we must consider the exact collision integral $I_{\nu_{\alpha}}$, that includes all relevant two-body weak reactions of the type $\nu_{\alpha}(1)+2 \rightarrow 3+4$ involving neutrinos and $e^{ \pm}$,

$$
\begin{aligned}
I_{\nu_{\alpha}} & {\left[f_{\nu_{e}}, f_{\nu_{\mu}}, f_{\nu_{\tau}}\right]=\frac{1}{2 E_{1}} \sum_{\text {reactions }} \int \frac{d^{3} p_{2}}{2 E_{2}(2 \pi)^{3}} \frac{d^{3} p_{3}}{2 E_{3}(2 \pi)^{3}} \frac{d^{3} p_{4}}{2 E_{4}(2 \pi)^{3}} } \\
& \times(2 \pi)^{4} \delta^{(4)}\left(p_{1}+p_{2}-p_{3}-p_{4}\right) F\left[\varrho_{\alpha \alpha}\left(p_{1}\right), f_{2}, f_{3}, f_{4}\right]\left|M_{12 \rightarrow 34}\right|^{2},
\end{aligned}
$$

Here $F \equiv f_{3} f_{4}\left(1-\varrho_{\alpha \alpha}\left(p_{1}\right)\right)\left(1-f_{2}\right)-\varrho_{\alpha \alpha}\left(p_{1}\right) f_{2}\left(1-f_{3}\right)\left(1-f_{4}\right)$ is the statistical factor (when the particle $i=2,3,4$ is a neutrino $\nu_{\beta}$ one substitutes $f_{i}$ with the corresponding diagonal term $\varrho_{\beta \beta}\left(p_{i}\right)$ ), and $M_{12 \rightarrow 34}$ is the process amplitude. In ref. [9] the complete list of relevant processes and corresponding squared amplitudes are reported, and it is shown that some of the integrals can be analytically performed, reducing $I_{\nu_{\alpha}}$ to a two-dimensional integral. We have actually checked that including the effect of mixing in the statistical terms of Eq. (7) as in [25] leads to very small modifications of our results (a similar conclusion was found in [19] with QREs and in the Boltzmann limit).

The kinetic equations for the neutrino density matrix are supplemented by the continuity equation for the total energy density $\rho_{\mathrm{R}}$,

$$
\frac{d \rho_{\mathrm{R}}}{d t}=-3 H\left(\rho_{\mathrm{R}}+P_{\mathrm{R}}\right)
$$

where $P_{\mathrm{R}}$ is the total pressure of the relativistic plasma: the three neutrino states and the electromagnetic components $\gamma$ and $e^{ \pm}$(always in equilibrium with temperature $T_{\gamma}$ ). This equation gives the evolution of the photon temperature $T_{\gamma}$. Finally, the finite temperature QED corrections to the electromagnetic plasma modify the equations of state of $e^{ \pm}$and $\gamma$ and are taken into account as described in [13].

$\overline{3}$ Here the density matrix $\varrho(\bar{\varrho})$ is the integrated neutrino (antineutrino) density matrix so that, for example, $\varrho_{e e}$ is the total number density of electron neutrinos. 


\subsection{Computation and technical issues}

Our set of Eqs. (4) and (8) are simplified when we use the following dimensionless variables instead of time, neutrino momenta and photon temperature:

$$
x \equiv m R, \quad y \equiv p R, \quad z \equiv T_{\gamma} R
$$

where $m$ is an arbitrary mass scale that we choose to be the electron mass and $R$ is the universe scale factor (normalized so that $R(t) \rightarrow 1 / T_{\gamma}$ at large temperatures). For details and the specific forms of the different terms in the equations, we refer the reader to the appendix in [2] (but note that in this work the mass scale $m$ was chosen to be $1 \mathrm{MeV}$ ).

The kinetic equations (4) are integro-differential due to the collision terms in Eq. (7). In previous works without neutrino oscillations, the system was solved either using a discretization in a grid of dimensionless momenta as in $[8,9]$ or with an expansion of the non-thermal distortions in moments as in $[11,13]$. Here we follow the discretization method, calculating the evolution of the neutrino density matrix on a grid of 100 neutrino momenta in the range $y_{i} \in[0.02,20]$.

We start to compute the evolution of the system at a value of the parameter $x_{\text {in }}=m_{e} /(10 \mathrm{MeV})$, when weak interactions were effective enough to keep neutrinos in equilibrium with the electromagnetic plasma. Therefore, the initial values ${ }^{4}$ of the components of the density matrix $\varrho\left(y_{i}, x\right)$ are either $\left[\exp \left(y_{i} / z_{\text {in }}\right)+1\right]^{-1}$ (diagonal components) or zero (off-diagonal), since flavour oscillations are suppressed at large temperatures by medium effects. Finally, the initial value of the dimensionless photon temperature is $z_{\text {in }}=1.00003$, which can be found solving Eq. (8) with neutrinos fully coupled [10].

The system of equations is solved from $x_{\text {in }}$ until a value of $x$ when both the neutrino distortions and the comoving photon temperature $z$ are frozen, approximately at $x_{\text {fin }} \simeq 35$. In order to solve simultaneously for the generation of the distortion and the effect of flavour oscillations we proceed as follows. First the change in the diagonal terms of $\varrho\left(y_{i}, x\right)$ from the collision integrals (neutrino heating) is found in a step $\Delta x$ whose value is set by the typical time scale of electron-positron annihilation rate (we use 1000 steps in $\log (x)$ in the range $\left.\left[x_{\mathrm{in}}, x_{\mathrm{fin}}\right]\right)$. Then the (fast) effect of the oscillations is then calculated by following the neutrino density matrix evolution on a smaller $x$ step, given by $\Delta x / 100$.

$\overline{4}$ Actually we solve the kinetic equations for the components of $\varrho\left(y_{i}, x\right)$ normalized to the FD distribution in Eq. (1). 


\section{Results}

We have numerically calculated the evolution of the neutrino density matrix solving the system of Eqs. (4) and (8), during the full process of neutrino decoupling. In order to compare with previous results, we have also calculated the case without neutrino mixing, with and without QED corrections. When flavour neutrino oscillations are included, we consider two cases, corresponding to the best-fit values of the mixing parameters in Eq. (6) and either $\theta_{13}=0$ or $s_{13}^{2}=0.047$.

\subsection{Evolution of the non-thermal distortions}

We present in Fig. 1 the evolution of the distortion of the neutrino distribution as a function of $x$ for a particular neutrino momentum $(y=10)$. In the absence of mixing, the evolution of $f_{\nu}$ has been described in previous analyses (see e.g. [9,11]). At large temperatures or $x \lesssim 0.2$, neutrinos are in good thermal contact with $e^{ \pm}$and their distributions only change keeping an equilibrium shape with the photon temperature $[\exp (y / z(x))+1]^{-1}$ (the $T_{\gamma}$ line in the figure). In the intermediate region $0.2 \lesssim x \lesssim 4$, weak interactions become less effective in a momentum-dependent way, leading to distortions in the neutrino spectra which are larger for $\nu_{e}$ 's than for the other flavours. Finally, at larger values of $x$ neutrino decoupling is complete and the distortions reach their asymptotic values. For the particular neutrino momentum in Fig. 1, the final value of the distribution is $4.4 \%\left(\nu_{e}\right)$ and $2 \%\left(\nu_{\mu, \tau}\right)$ larger than in the instantaneous decoupling limit.

It is obvious that flavour neutrino oscillations will modify the generation of neutrino distortions if they are effective at the relevant range of temperatures. This depends on the different terms in the kinetic equations for the neutrino density matrix, in particular the relative importance of the oscillation term (of order $\Delta m^{2} / 2 p$ ) which grows as $x^{2}$, with respect to the background potential proportional to the energy density of electrons and positrons (decreases as $x^{4}$ ), since the other charged leptons have already disappeared. In the range $x \lesssim 0.3$ the refractive term dominates, suppressing flavour oscillations so that the neutrino distributions grow as in the absence of mixing. Then the $e^{ \pm}$potential adiabatically disappears, leading to the usual MSW-type evolution and a convergence of the flavour neutrino distortions. Finally, the oscillation term dominates and oscillations proceed as in vacuum (in an expanding universe, as calculated in [28]). As can be seen for example in Fig. 1, if $\theta_{13}=0$ the final value of the distribution of $\nu_{e}$ 's at $y=10$ is reduced to $3.4 \%$ while for $\nu_{\mu, \tau}$ increases to $2.4 \%$. When we take $s_{13}^{2}=0.047$, we find $3.2 \%$ for $\nu_{e}$ 's and a different distortion for $\nu_{\mu}$ 's $(2.6 \%)$ and $\nu_{\tau}$ 's $(2.4 \%)$. 


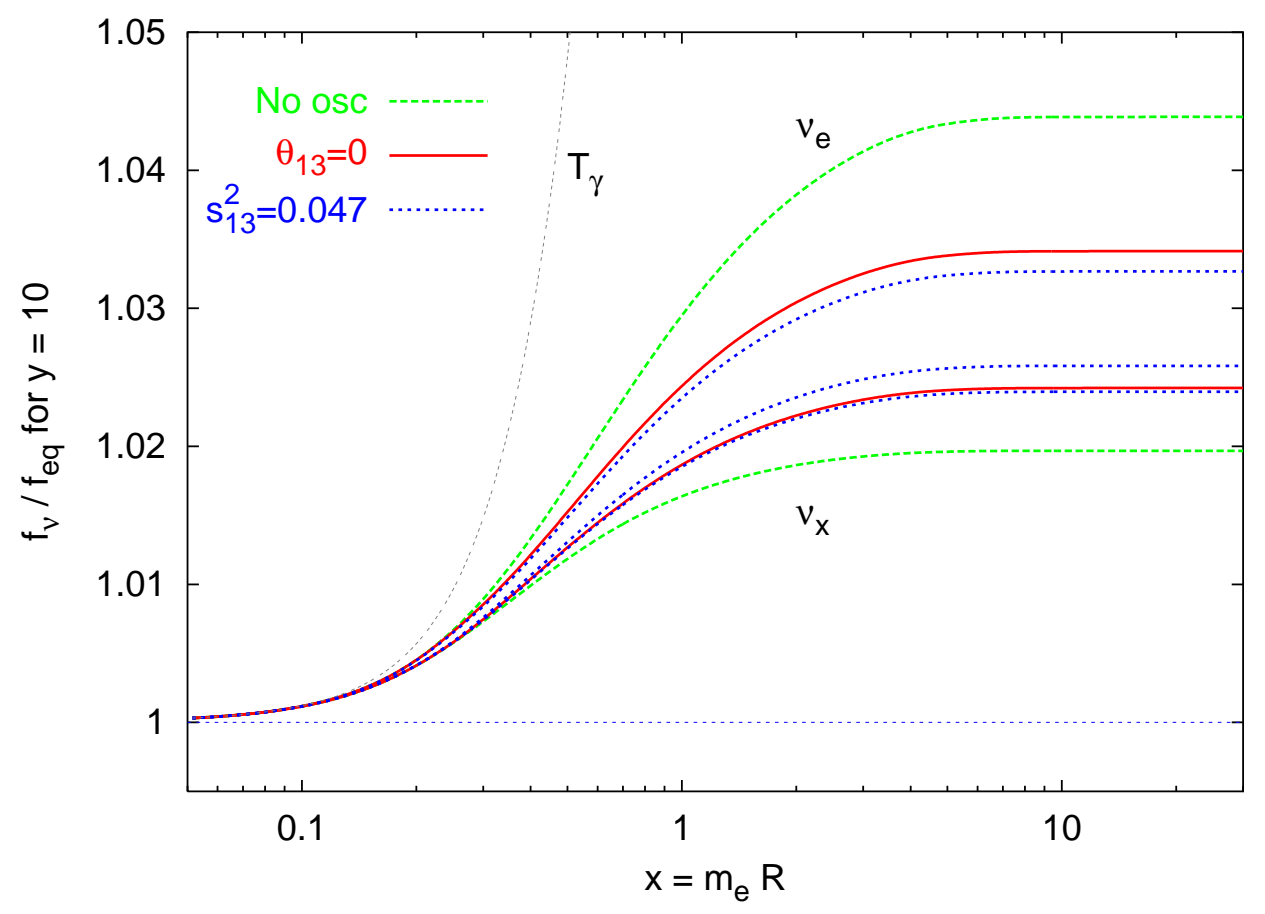

Fig. 1. Evolution of the distortion of the $\nu_{e}$ and $\nu_{x}=\nu_{\mu, \tau}$ spectrum for a particular comoving momentum $(y=10)$. In the case with $\theta_{13} \neq 0$ one can distinguish the distortions for $\nu_{\mu}$ (middle line) and $\nu_{\tau}$ (lower line). The line labeled with $T_{\gamma}$ corresponds to the distribution of a neutrino in full thermal contact with the electromagnetic plasma.

For a more detailed description of the evolution of flavour oscillations at this epoch, we refer the reader to [2].

\subsection{Frozen spectra and $N_{\text {eff }}$}

We show in Fig. 2 the asymptotic values of the flavour neutrino distribution, for the cases without oscillations and with non-zero mixing. The dependence of the non-thermal distortions in momentum is well visible, which reflects the fact that more energetic neutrinos were interacting with $e^{ \pm}$for a longer period. Moreover, the effect of neutrino oscillations is evident, reducing the difference between the flavour neutrino distortions.

Once we have found the final neutrino distributions, the frozen values of some quantities characterizing neutrino heating can be calculated. In Tables 1 and 2 we present our results for the dimensionless photon temperature $z_{\text {fin }}$, the change in the neutrino energy densities with respect to $\rho_{\nu_{0}}$ (the energy density in the instantaneous decoupling limit) and the asymptotic effective number of 


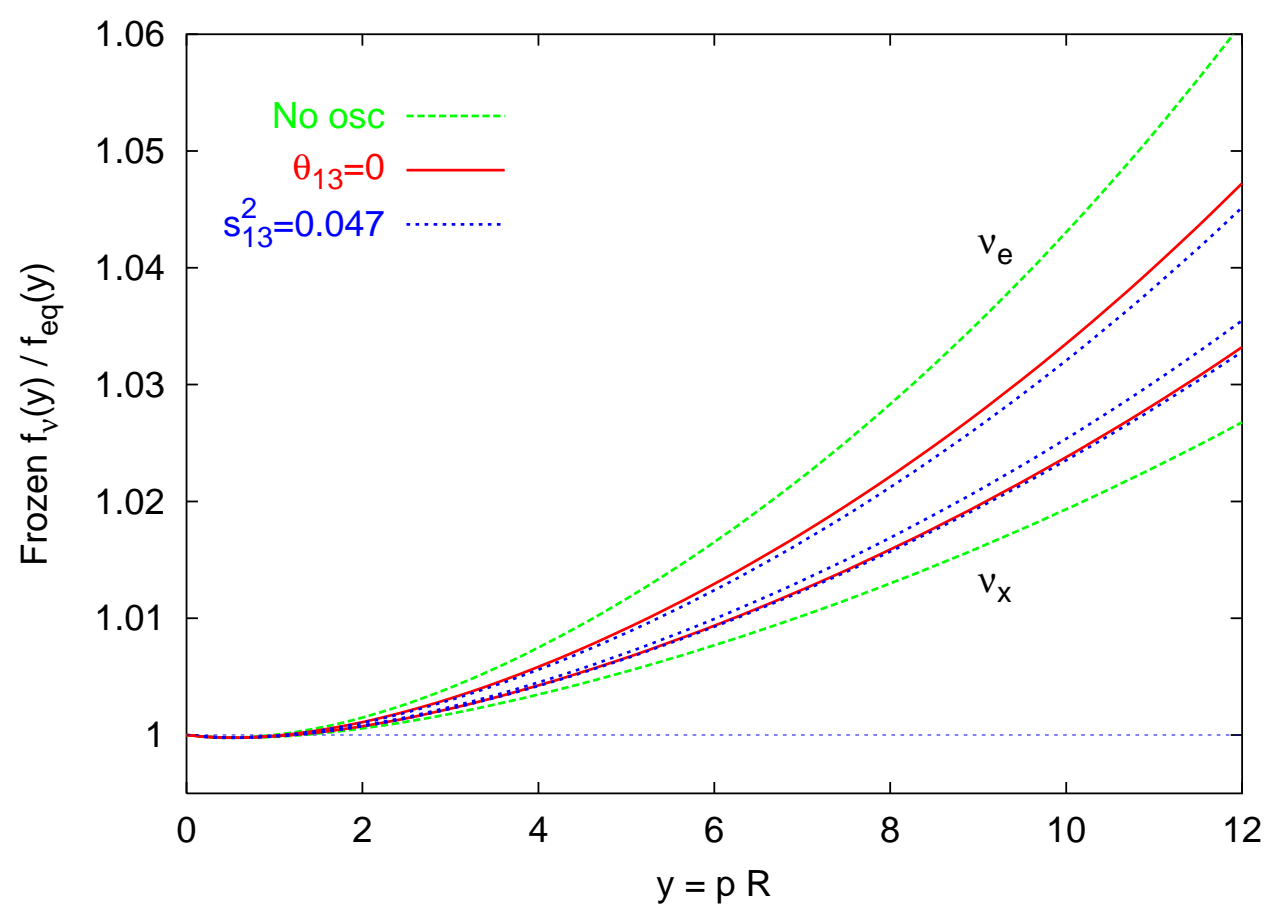

Fig. 2. Frozen distortions of the flavour neutrino spectra as a function of the comoving momentum. In the case with $\theta_{13} \neq 0$ one can distinguish the distortions for $\nu_{\mu}$ (middle line) and $\nu_{\tau}$ (lower line).

neutrinos $N_{\text {eff }}$ as defined in Eq. (2), that can be calculated as

$$
N_{\mathrm{eff}}=\left(\frac{z_{0}}{z_{\mathrm{fin}}}\right)^{4}\left(3+\frac{\delta \rho_{\nu_{e}}}{\rho_{\nu_{0}}}+\frac{\delta \rho_{\nu_{\mu}}}{\rho_{\nu_{0}}}+\frac{\delta \rho_{\nu_{\tau}}}{\rho_{\nu_{0}}}\right)
$$

where $z_{0}=(11 / 4)^{1 / 3} \simeq 1.40102$. Note that one can not relate $z_{\text {fin }}$ with a ratio of photon and neutrino temperatures, since the neutrino spectra are non-thermal and strictly speaking $T_{\nu}$ is not defined.

In the absence of mixing, our results in Table 1 without QED corrections agree with previous works $[9,11]$, while including QED corrections we find a slightly smaller $z_{\text {fin }}$ than [13] that is due to a more accurate numerical calculation of the evolution of $z(x)$. After evaluating the precision in the numerical calculations (modifying our choice for the grid in neutrino momenta, the initial value of $x$, etc), we estimate that the accuracy in the values of $N_{\text {eff }}$ is \pm 0.002 . For comparison, we also show the results of two toy cases where all neutrino flavours have the same interactions with $e^{+}-e^{-}$as $\nu_{e}$ 's or $\nu_{\mu}$ 's, respectively.

When flavour oscillations are taken into account, our results in Table 2 show that, while the modifications in the individual values of $\rho_{\nu_{\alpha}}$ can be clearly seen, the contribution of neutrino heating to the total relativistic energy density is almost unchanged, with a value of $N_{\text {eff }}=3.046$. The difference with respect to 
Table 1

Frozen values of $z_{\text {fin }}$, the neutrino energy densities $\delta \bar{\rho}_{\nu_{\alpha}} \equiv \delta \rho_{\nu_{\alpha}} / \rho_{\nu_{0}}, N_{\text {eff }}$ and $\Delta Y_{p}$ in the absence of flavour neutrino mixing.

\begin{tabular}{lccccc}
\hline Case & $z_{\text {fin }}$ & $\delta \bar{\rho}_{\nu_{e}}$ & $\delta \bar{\rho}_{\nu_{\mu, \tau}}$ & $N_{\text {eff }}$ & $\Delta Y_{p}$ \\
\hline No mixing & 1.3978 & $0.94 \%$ & $0.43 \%$ & 3.046 & $1.71 \times 10^{-4}$ \\
\hline No mixing (no QED) & 1.3990 & $0.95 \%$ & $0.43 \%$ & 3.035 & $1.47 \times 10^{-4}$ \\
No mixing (all $\left.\nu_{e}\right)$ & 1.3966 & $0.95 \%$ & $0.95 \%$ & 3.066 & $3.57 \times 10^{-4}$ \\
No mixing (all $\left.\nu_{\mu}\right)$ & 1.3986 & $0.35 \%$ & $0.35 \%$ & 3.031 & $1.35 \times 10^{-4}$ \\
\hline
\end{tabular}

Table 2

Frozen values of $z_{\text {fin }}$, the neutrino energy densities $\delta \bar{\rho}_{\nu_{\alpha}} \equiv \delta \rho_{\nu_{\alpha}} / \rho_{\nu_{0}}, N_{\text {eff }}$ and $\Delta Y_{p}$ including flavour neutrino oscillations.

\begin{tabular}{lcccccc}
\hline Case & $z_{\text {fin }}$ & $\delta \bar{\rho}_{\nu_{e}}$ & $\delta \bar{\rho}_{\nu_{\mu}}$ & $\delta \bar{\rho}_{\nu_{\tau}}$ & $N_{\text {eff }}$ & $\Delta Y_{p}$ \\
\hline$\theta_{13}=0$ & 1.3978 & $0.73 \%$ & $0.52 \%$ & $0.52 \%$ & 3.046 & $2.07 \times 10^{-4}$ \\
$\sin ^{2} \theta_{13}=0.047$ & 1.3978 & $0.70 \%$ & $0.56 \%$ & $0.52 \%$ & 3.046 & $2.12 \times 10^{-4}$ \\
\hline Bimaximal $\left(\theta_{13}=0\right)$ & 1.3978 & $0.69 \%$ & $0.54 \%$ & $0.54 \%$ & 3.045 & $2.13 \times 10^{-4}$ \\
\hline
\end{tabular}

the unmixed case is only seen in the results within the following decimal place: 3.0458 (no oscillations) to 3.0455 (with oscillations, either $s_{13}^{2}=0$ or 0.047 ). We checked that even in the case of bimaximal mixing where $\theta_{12}=\theta_{23}=\pi / 4$ and $\theta_{13}=0$ (disfavoured by present experimental data) the change in $N_{\text {eff }}$ is minimal, with a very small decrease to 3.0454. Thus the presence of neutrino oscillations leads to slightly less efficient neutrino heating ${ }^{5}$. Our findings are therefore quite different than those presented in [19], where a very small but positive change in the neutrino energy density with respect to the unmixed case was found. This difference is probably due to the approximations used in that paper.

The effect of neutrino heating on any quantity that characterizes relic neutrinos is found replacing the Fermi-Dirac distribution with the spectra as given in Fig. 2. Only when neutrinos are still relativistic one finds an integrated effect of the distortion. For instance, the contribution of relativistic relic neutrinos to the total energy density is taken into account just by using $N_{\text {eff }}=3.046$. But in general, for numerical calculations such as those done by the codes CMBFAST [30] or CAMB [31], one must include the distortions as a function of neutrino momenta. Our results for the case with flavour oscillations and $\theta_{13}=0$ (the red lines in Fig. 2) are very well reproduced by the analytical fits

$$
f_{\nu_{e}}(y)=f_{\mathrm{eq}}(y)\left[1+10^{-4}\left(1-2.2 y+4.1 y^{2}-0.047 y^{3}\right)\right]
$$

5 An analysis of neutrino heating in presence of non-standard neutrino interactions is presently under study [29]. 


$$
f_{\nu_{\mu, \tau}}(y)=f_{\mathrm{eq}}(y)\left[1+10^{-4}\left(-4+2.1 y+2.4 y^{2}-0.019 y^{3}\right)\right]
$$

Note, however, that for any cosmological epoch when neutrino masses can be relevant one must consider the neutrino mass eigenstates $\nu_{1,2,3}$. The corresponding momentum distributions can be easily found from the flavour ones through the relation

$$
f_{\nu_{i}}(y)=\sum_{\alpha=e, \mu, \tau}\left|U_{\alpha i}\right|^{2} f_{\nu_{\alpha}}(y)
$$

which, in the case with oscillations and $\theta_{13}=0$ gives the simple relations

$$
\begin{aligned}
& f_{\nu_{1}}(y)=0.7 f_{\nu_{e}}(y)+0.3 f_{\nu_{x}}(y) \\
& f_{\nu_{2}}(y)=0.3 f_{\nu_{e}}(y)+0.7 f_{\nu_{x}}(y) \\
& f_{\nu_{3}}(y)=f_{\nu_{x}}(y)
\end{aligned}
$$

where we have used that $f_{\nu_{x}}=f_{\nu_{\mu}}=f_{\nu_{\tau}}$.

Finally, let us consider the contribution of massive neutrinos to the present value of the energy density of the Universe. In general, this must be numerically evaluated for any choice of neutrino masses $\left(m_{1}, m_{2}, m_{3}\right)$ using the distorted distributions described above. However, in the particular case when neutrino masses are almost degenerate it is easy to find, using the expressions in Eqs. (11) or (13), that the contribution of neutrinos in units of the critical value of the energy density is

$$
\Omega_{\nu}=\frac{\rho_{\nu}}{\rho_{c}}=\frac{3 m_{0}}{93.14 h^{2} \mathrm{eV}}
$$

where $h$ is the present value of the Hubble parameter in units of $100 \mathrm{~km} \mathrm{~s}^{-1}$ $\mathrm{Mpc}^{-1}$ and $m_{0}$ is the neutrino mass scale. Here the number in the denominator is slightly smaller than in the instantaneous decoupling limit (94.12).

\subsection{Primordial Nucleosynthesis}

Let us now discuss the effects of neutrino heating on BBN, and in particular on the production of primordial ${ }^{4} \mathrm{He}$. Neglecting neutrino oscillations, it is well known that the non-thermal neutrino distortions change the prediction of the primordial ${ }^{4} \mathrm{He}$ mass fraction $Y_{p}$ by a small amount, of the order $[5,6,7,8,9,10,11]$

$$
\Delta Y_{p} \simeq 1.5 \times 10^{-4}
$$


One would then naively guess that neutrino oscillations, as a sub-leading modification, can only marginally change this effect. However, the small number in Eq. (15) comes out from subtle cancellations of much larger effects, each one responsible of changes in $Y_{p}$ of $\mathcal{O}\left(10^{-3}\right)$ (see e.g. [5]). If neutrino oscillations break these accidental cancellations, the effect could be comparable with that of Eq. (15). Indeed, this seems to be suggested by the approximate analysis performed in [19], where effects up to $\delta Y_{p} \approx(1.1-1.3) \times 10^{-4}$ were found (here we use $\Delta Y_{p}$ for the total change due to neutrino heating and $\delta Y_{p}$ to the net effect induced by neutrino oscillations).

In view of the results discussed in the previous sections, however, where appreciable differences with respect to the picture presented in [19] are found, the oscillation-induced $\delta Y_{p}$ is likely to change. Before giving our numerical results, we give a simple estimate of the expected effects. According to the arguments of $[5,7]$, in the approximation of thermal-equivalent distortions one can perform a perturbative estimate of the effects of neutrino reheating on the final neutron fraction $X_{n}$. In a framework where the scale factor $R=T_{\nu_{0}}^{-1}$ is kept fixed (i.e. unperturbed), one can identify three changes to $X_{n}$ :

(1) a change in the weak rates due to the distortion to $\nu_{e}-\bar{\nu}_{e}$ spectra, given by

$$
\delta X_{n}^{\nu} \approx-0.1 \frac{\delta T_{\nu_{e}}}{T_{\nu_{e}}} \approx-\frac{0.1}{4} \frac{\delta \rho_{\nu_{e}}}{\rho_{\nu_{e}}}
$$

(2) since the energy must be conserved, the overall extra energy density $\delta \rho_{\nu}$ is compensated by a decrease of the electromagnetic plasma contribution $\delta \rho_{\text {e.m. }}=-\delta \rho_{\nu}$ with respect to the instantaneous decoupling case. This changes in turn the weak rates (where the $e^{ \pm}$distributions enter), finally producing

$$
\delta X_{n}^{\mathrm{e} . \mathrm{m} .} \approx-0.1 \frac{\delta T_{\gamma}}{T_{\gamma}} \approx+\frac{0.1}{4} \frac{\delta \rho_{\nu}}{\rho_{\nu}}
$$

(3) given the high photon entropy, the BBN can start via the deuterium production $p+n \rightarrow \gamma+d$ only when the universe has cooled down to a temperature $T_{\mathrm{BBN}} \approx 0.07 \mathrm{MeV}$. At this point, the decay of free neutrons (practically the only weak process since the $n-p$ freezing at $T_{\mathrm{F}} \approx 0.7 \mathrm{MeV}$ ) stops and most of the neutrons are eventually fixed into ${ }^{4} \mathrm{He}$ nuclei. In formulae,

$$
X_{n}\left(T_{\mathrm{BBN}}\right)=X_{n}\left(T_{\mathrm{F}}\right) e^{-\left(t\left(T_{\mathrm{BBN}}\right)-t\left(T_{\mathrm{F}}\right)\right) / \tau_{n}},
$$

where $\tau_{n}$ is the neutron lifetime. The neutrino reheating changes the timetemperature relationship, thus the electromagnetic plasma reaches the value $T_{\mathrm{BBN}}$ at a different time given by [5]

$$
\frac{\delta t_{\mathrm{BBN}}}{t_{\mathrm{BBN}}} \approx-\frac{\delta \rho_{\nu}}{\rho_{\text {tot }}} \approx-\frac{1}{2} \frac{\delta \rho_{\nu}}{\rho_{\nu}}
$$


From Eqs. (18) and (19) one easily derives the approximate result

$$
\delta X_{n}^{t} \propto \frac{\delta \rho_{\nu}}{\rho_{\nu}} .
$$

Once using $Y_{p} \approx 1.33 X_{n}$ [5] and fixing the constant in Eq. (20) in order to get the result given in [19] for the no-oscillation case $\left(\Delta Y_{p}=1.2 \times 10^{-4}\right)$ one can predict the value of $\delta Y_{p}$ starting from $\delta \rho_{\nu_{e}}$ and $\delta \rho_{\nu}$. This simple approach gives $\delta Y_{p}^{\text {osc }} \approx 1.3 \times 10^{-4}$ (and $\delta Y_{p}^{M a x} \approx 1.6 \times 10^{-4}$ for the maximal mixing case), quite in nice agreement indeed with the numerical findings of [19] which are respectively 1.1 and $1.3 \times 10^{-4}$. This is obviously not unexpected, given the thermal-equivalent approximation used.

When applied to our findings, the same formula, with the same normalization to the data of [19], would predict changes of 0.66 and $0.76 \times 10^{-4}$ for the $\theta_{13}=0$ and $s_{13}^{2}=0.047$ cases, respectively. The main conclusion from this simple argument is that the variation $\delta Y_{p}$ induced by neutrino oscillations is likely to be smaller than what found in [19].

To improve our previous estimates, we have modified the BBN code developed in the past decade by the Naples group (see refs. $[16,17]$ ). Notice that $\bar{z} \equiv$ $m_{e} / T_{\gamma}=x / z$ is used there as independent variable. This implies that the neutrino heating effects previously described have to be re-interpreted in such a framework (see Appendix 3 of ref. [5] for an account of this issue). By definition there is no perturbation to the e.m. fluid; instead, the neutrino fluid is not only distorted by reheating, but also gets a correction from the modified relation $T_{\nu}-T_{\gamma}$ or equivalently $R(\bar{z})$. Finally, at a fixed $\bar{z}$, the Hubble function is obviously altered by the extra energy density due to neutrino distortions, and the time-temperature equation also gets a further correction piece which was called $N(\bar{z})$ in ref. [17]. Fixing as a background the spectra where reheating as well as QED effects were taken into account, we calculated $\delta Y_{p}$ as follows. Both $\rho_{\nu}(\bar{z}), N(\bar{z})$, and the weak rates in the Born approximation were numerically evaluated for the case under consideration and the background solution. The differences between the two cases were treated as perturbations (keeping fixed the $\bar{z}$ of background) and then properly fitted. The use of the Born rates to calculate these extra terms does not constitute a bad approximation, since the effects we are dealing with are really tiny, and higher order corrections as those coming from radiative processes can be safely neglected.

In Tables 1 and 2 we also include our results for the change in $Y_{p}$. Our result for the standard reheating effect, $\Delta Y_{p}=1.71 \times 10^{-4}$, essentially agrees with the evaluation $\Delta Y_{p} \simeq 1.5 \times 10^{-4}$ present in literature (see e.g. [7]), where the slightly larger value arises from a larger $N_{\text {eff }}$ from QED corrections. In [7] it was noted that the effect on $Y_{p}$ could be reproduced by an effective increase $\Delta N_{\mathrm{BBN}}$ in the number of neutrinos of about 0.01 (actually $\simeq 0.013$, for our findings). Although academic on the light of present observational accuracies, 
Table 3

Comparison of the exact BBN results with the $\Delta N_{\mathrm{BBN}}$ approximation.

\begin{tabular}{lll}
\hline Nuclide & Exact & $\Delta N_{\mathrm{BBN}}=0.013$ \\
\hline$\Delta Y_{p}$ & $1.71 \times 10^{-4}$ & $1.76 \times 10^{-4}$ \\
\hline$\Delta\left({ }^{2} \mathrm{H} / \mathrm{H}\right)$ & $-0.0068 \times 10^{-5}$ & $+0.0044 \times 10^{-5}$ \\
\hline$\Delta\left({ }^{3} \mathrm{He} / \mathrm{H}\right)$ & $-0.0011 \times 10^{-5}$ & $+0.0007 \times 10^{-5}$ \\
\hline$\Delta\left({ }^{7} \mathrm{Li} / \mathrm{H}\right)$ & $+0.0214 \times 10^{-10}$ & $-0.0058 \times 10^{-10}$ \\
\hline
\end{tabular}

we warn the reader that this approximation only works for ${ }^{4} \mathrm{He}$. Indeed for the other relevant nuclides it produces a change at the $\mathcal{O}(0.1 \%)$ level that is exactly in the opposite direction of the true one, as shown in Table 3.

The effects of the oscillations on $\Delta Y_{p}$ in Table 2 can be easily explained qualitatively. A decrease of $\delta \rho_{\nu_{e}} / \rho_{\nu_{0}}$, which is the unavoidable consequence of neutrino oscillations, leads to an increase in $Y_{p}$ (see Eq. (16)). On the other hand, a decrease of $\delta \rho_{\nu} / \rho_{\nu_{0}}$ causes a decrease of $Y_{p}$ (see Eq. (17)). This is in fact what we find, so that the approximate cancellation of the effects (1) and (2) still holds, differently than what described in [19], leaving a sub-leading contribution of the order of few $\times 10^{-5}$ as the effect of neutrino oscillations on $Y_{p}$. Modifying the neutrino mixing parameters only leads to even smaller effects, since $\Delta Y_{p}$ changes from $2.07 \times 10^{-4}\left(\theta_{13}=0\right)$ to $2.12 \times 10^{-4}\left(s_{13}^{2}=0.047\right)$. All the previous results were obtained for a baryon fraction $\omega_{b}=0.023$, in agreement with the WMAP determination [32]. As already reported in [7], the effects show only a weak dependency on the exact value of $\omega_{b}$, for a large interval of values of this parameter. In particular, in the range $\omega_{b}=0.020-0.026$ the changes in the absolute values reported in Tables 1 and 2 are of $\mathcal{O}(1 \%)$, while the relative values are practically unchanged.

In summary, we find that the global change $\Delta Y_{p} \simeq 2.1 \times 10^{-4}$ agrees with the results in [19] because of the inclusion of QED effects, but the net effect due to oscillations is about a factor 3 smaller than what previously estimated. We think that the main reason of the discrepancy is due to the failure of the momentum-averaged approximation to reproduce the true distortions.

\section{Conclusions}

In this paper we have performed a new analysis of the effect of flavour oscillations on the neutrino decoupling phase in the early Universe. By numerically solving the relevant kinetic equations we have found the evolution of the distortions on the energy distributions of neutrinos caused by residual interactions with the electromagnetic plasma during the electron/positron annihilation 
phase.

The inclusion of neutrino oscillations modifies the evolution and final values of the distortions in the flavour basis, reducing that of $\nu_{e}$ 's and enhancing those of the other neutrino flavours. We have calculated the frozen values of the neutrino distributions, that should be used in any numerical evaluation of quantities related to relic neutrinos, like those done in codes such as CMBFAST or CAMB. In particular, we find that that the asymptotic value of the total neutrino energy density in presence of flavour oscillations is essentially unchanged with respect to the unmixed case, being parametrized by an effective number of neutrinos $N_{\text {eff }}=3.046$. In addition, we found the effect of neutrino heating on the products of BBN, which in the case of ${ }^{4} \mathrm{He}$ is approximately $20 \%$ larger when flavour oscillations are included.

Present bounds on the radiation content of the Universe from CMB and LSS data, of the order $N_{\text {eff }}<7(95 \% \mathrm{CL})$, are still far from the effect caused by neutrino heating. But a value of $\Delta N_{\text {eff }}=0.046$ would be close to the potential sensitivity of future CMB data from PLANCK, according to the forecast analysis in ref. [35]. More recent analyses in refs. [36,37] show that this conclusion was too optimistic, reducing the sensitivity to $\Delta N_{\text {eff }} \sim 0.2$.

Finally, a few words on the detectability of the shift on BBN products caused by neutrino heating. For $Y_{p}$, the effect of the reheating is below the $\mathcal{O}(0.1 \%)$ level, of which only about $0.02 \%$ are due to flavour oscillations. The theoretical uncertainty is at least of $0.2 \%$, while the observational error is likely to be $\approx 5 \%$ and dominated by systematics, with statistical errors at least at the $1 \%$ level (see e.g. [17] and references therein). Obviously there is no hope to appreciate such tiny effects: moreover, given the theoretical uncertainties, an absolute prediction of the ${ }^{4} \mathrm{He}$ yield at the $0.01 \%$ level would imply a significant improvement in many sub-leading aspects of the BBN physics and numerics, which is clearly unjustified given the existing much larger observational uncertainties. The overall change due to the reheating in the other nuclides yields is at most of $\mathcal{O}(0.1 \%)$, and the net effect due to oscillations practically negligible. Once considered that the existing (theoretical as well as observational) uncertainties are not better than $\mathcal{O}(10-20 \%)$ for the case of deuterium, one has to conclude that definitively one cannot gain information on the standard scenario of neutrino oscillations physics from BBN.

\section{Acknowledgments}

This work was supported by a Spanish-Italian AI, the Spanish grant BFM200200345, as well as a CICYT-INFN agreement. SP was supported by a Ramón y Cajal contract of MEC. In Munich, this work was supported in part by the 
Deutsche Forschungsgemeinschaft under grant SFB 375.

\section{References}

[1] E.W. Kolb and M.S. Turner, The Early Universe (Addison-Wesley, 1990).

[2] A.D. Dolgov et al, Nucl. Phys. B 632 (2002) 363 hep-ph/0201287.

[3] D.A. Dicus et al, Phys. Rev. D 26 (1982) 2694.

[4] A.D. Dolgov, Phys. Rept. 370 (2002) 333 hep-ph/0202122.

[5] S. Dodelson and M.S. Turner, Phys. Rev. D 46 (1992) 3372.

[6] A.D. Dolgov and M. Fukugita, Phys. Rev. D 46 (1992) 5378.

[7] B.D. Fields, S. Dodelson and M.S. Turner, Phys. Rev. D 47 (1993) 4309 astro-ph/9210007.

[8] S. Hannestad and J. Madsen, Phys. Rev. D 52 (1995) 1764 astro-ph/9506015.

[9] A.D. Dolgov, S.H. Hansen and D.V. Semikoz, Nucl. Phys. B 503 (1997) 426 hep-ph/9703315.

[10] A.D. Dolgov, S.H. Hansen and D.V. Semikoz, Nucl. Phys. B 543 (1999) 269 hep-ph/9805467.

[11] S. Esposito et al, Nucl. Phys. B 590 (2000) 539 astro-ph/0005573.

[12] N. Fornengo, C.W. Kim and J. Song, Phys. Rev. D 56 (1997) 5123 hep-ph/9702324.

[13] G. Mangano, G. Miele, S. Pastor and M. Peloso, Phys. Lett. B 534 (2002) 8 astro-ph/0111408.

[14] V.F. Shvartsman, Pisma Zh. Eksp. Teor. Fiz. 9 (1969) 315 [JETP Lett. 9 (1969) 184].

[15] G. Steigman, D.N. Schramm and J.R. Gunn, Phys. Lett. B 66 (1977) 202.

[16] A. Cuoco et al, Int. J. Mod. Phys. A 19 (2004) 4431 astro-ph/0307213.

[17] P.D. Serpico et al, JCAP 0412 (2004) 010 astro-ph/0408076.

[18] P. Langacker, S.T. Petcov, G. Steigman and S. Toshev, Nucl. Phys. B 282 (1987) 589.

[19] S. Hannestad, Phys. Rev. D 65 (2002) 083006 astro-ph/0111423.

[20] K. Ichikawa, M. Kawasaki and F. Takahashi, astro-ph/0505395.

[21] P. Di Bari, Phys. Rev. D 65 (2002) 043509 hep-ph/0108182. 
[22] K.N. Abazajian, Astropart. Phys. 19 (2003) 303 astro-ph/0205238.

[23] D. Kirilova, Int. J. Mod. Phys. D 13 (2004) 831 hep-ph/0209104.

[24] M. Cirelli, G. Marandella, A. Strumia and F. Vissani, Nucl. Phys. B 708 (2005) 215 hep-ph/0403158.

[25] G. Sigl and G. Raffelt, Nucl. Phys. B 406 (1993) 423.

[26] B.H. McKellar and M.J. Thomson, Phys. Rev. D 49 (1994) 2710.

[27] M. Maltoni, T. Schwetz, M.A. Tórtola and J.W.F. Valle, New J. Phys. 6 (2004) 122 hep-ph/0405172.

[28] D.P. Kirilova, preprint JINR-E2-88-301.

[29] T. Pinto et al, in preparation.

[30] U. Seljak and M. Zaldarriaga, Astrophys. J. 469 (1996) 437 astro-ph/9603033. See also the webpage http://cmbfast.org

[31] A. Lewis, A. Challinor and A. Lasenby, Astrophys. J. 538 (2000) 473 astro-ph/9911177. See also the webpage http://camb.info

[32] D.N. Spergel et al [WMAP Collaboration], Astrophys. J. Suppl. 148, 175 (2003) astro-ph/0302209.

[33] S. Hannestad and G. Raffelt, JCAP 0404 (2004) 008 hep-ph/0312154.

[34] P. Crotty, J. Lesgourgues and S. Pastor, Phys. Rev. D 69 (2004) 123007 hep-ph/0402049.

[35] R.E. Lopez, S. Dodelson, A. Heckler and M.S. Turner, Phys. Rev. Lett. 82 (1999) 3952 astro-ph/9803095.

[36] R. Bowen et al, Mon. Not. Roy. Astron. Soc. 334 (2002) 760 astro-ph/0110636.

[37] S. Bashinsky and U. Seljak, Phys. Rev. D 69 (2004) 083002 astro-ph/0310198. 